\title{
Advancements in the Development of a Directional-Position Sensing Fast Neutron Detector using Acoustically Tensioned Metastable Fluids
}

Brian C. Archambault ${ }^{1}$, Jeffrey A. Webster ${ }^{2}$, Thomas F. Grimes ${ }^{2}$, Kevin Fischer ${ }^{2}$, Alex R. Hagen ${ }^{2}$, and Rusi P. Taleyakhan ${ }^{1,2}$

${ }^{1}$ Sagamore Adams Laboratories, Lafayette, Indiana, USA

${ }^{2}$ Purdue University, West Lafayette, Indiana, USA

Corresponding author: rusi@purdue.edu

1 Abstract-Advancements in the development of a direction and position sensing fast neutron detector which

2 utilizes the directional acoustic tensioned metastable fluid detector (D-ATMFD) are described. The

3 resulting D-ATMFD sensor is capable of determining the direction of neutron radiation with a single

4 compact detector versus use of arrays of detectors in conventional directional systems. Directional neutron

5 detection and source positioning offer enhanced detection speeds in comparison to traditional proximity

6 searching; including enabling determination of the neutron source shape, size, and strength in near real time.

7 This paper discusses advancements that provide the accuracy and precision of ascertaining directionality and

8 source localization information utilizing enhanced signal processing-cum-signal analysis, refined

9 computational algorithms, and on-demand enlargement capability of the detector sensitive volume. These

10 advancements were accomplished utilizing experimentation and theoretical modeling. Benchmarking and

11 qualifications studies were successfully conducted with random and fission based special nuclear material

12 (SNM) neutron sources $\left({ }^{239} \mathrm{Pu}-\mathrm{Be}\right.$ and $\left.{ }^{252} \mathrm{Cf}\right)$. These results of assessments have indicated that the $\mathrm{D}-$

13 ATMFD compares well in technical performance with banks of competing directional fast neutron detector

technologies under development worldwide, but it does so with: a single detector unit; an unlimited field of

view; and at a significant reduction in both cost and size while remaining completely blind to common

background (e.g., beta-gamma) radiation. Rapid and direct SNM neutron source imaging with two D-

ATMFD sensors was experimentally demonstrated, and furthermore, validated via multidimensional nuclear 
particle transport simulations utilizing MCNP-PoliMi. Characterization of a scaled D-ATMFD based radiation portal monitor (RPM) as a cost-effective and efficient ${ }^{3} \mathrm{He}$ sensor replacement was performed utilizing MCNP-PoliMi simulations, the results of which are discussed and presented.

\section{Introduction}

The development of directional neutron detectors for SNM is a relatively new field, garnering significant interest over the last decade. Directional fast neutron detectors have a number of potential applications, including locating and monitoring of SNM at nuclear facilities under safeguards regimes and the detection of sources of fast neutrons (including SNMs) in containers and packages. Examples of some of the state-of-theart directional fast neutron detector systems undergoing development include time projection chambers (TPC) [1][2], neutron scatter cameras [3][4][5], neutron imaging telescopes (FNIT) [6][7][8], and coded aperture cameras [9][10][11]. Development of the directional abilities of the ATMFD system represents a significant advancement to the current state-of-the-art directional neutron detectors. A single D-ATMFD system is capable of the directional detection of neutrons in a single portable detector with an unlimited field of view $(4 \pi)[12]$ and a significant reduction in size while remaining completely blind to non-neutron background [13]. This is accomplished with the potential for a significant cost reduction over comparable systems (e.g. \$500 - \$1K for D-ATMFD vs. $\$ 100 \mathrm{~K}$ - > \$300K for state-of-art directional systems). Advances in the ongoing scientific development and validation of the unique directional-cum-imaging capabilities of the D-ATMFD are the focus of this report.

\section{The D-ATMFD Sensor System -Overview}

The D-ATMFD used in this report is similar to the one previously used [14] with the exception that the top acoustic reflector is affixed directly to the top of the resonance chamber. Affixing the top reflector increased operational stability by avoiding sloshing of the top reflector and the detector fluid allowing the 
41 shown in Figure 1.

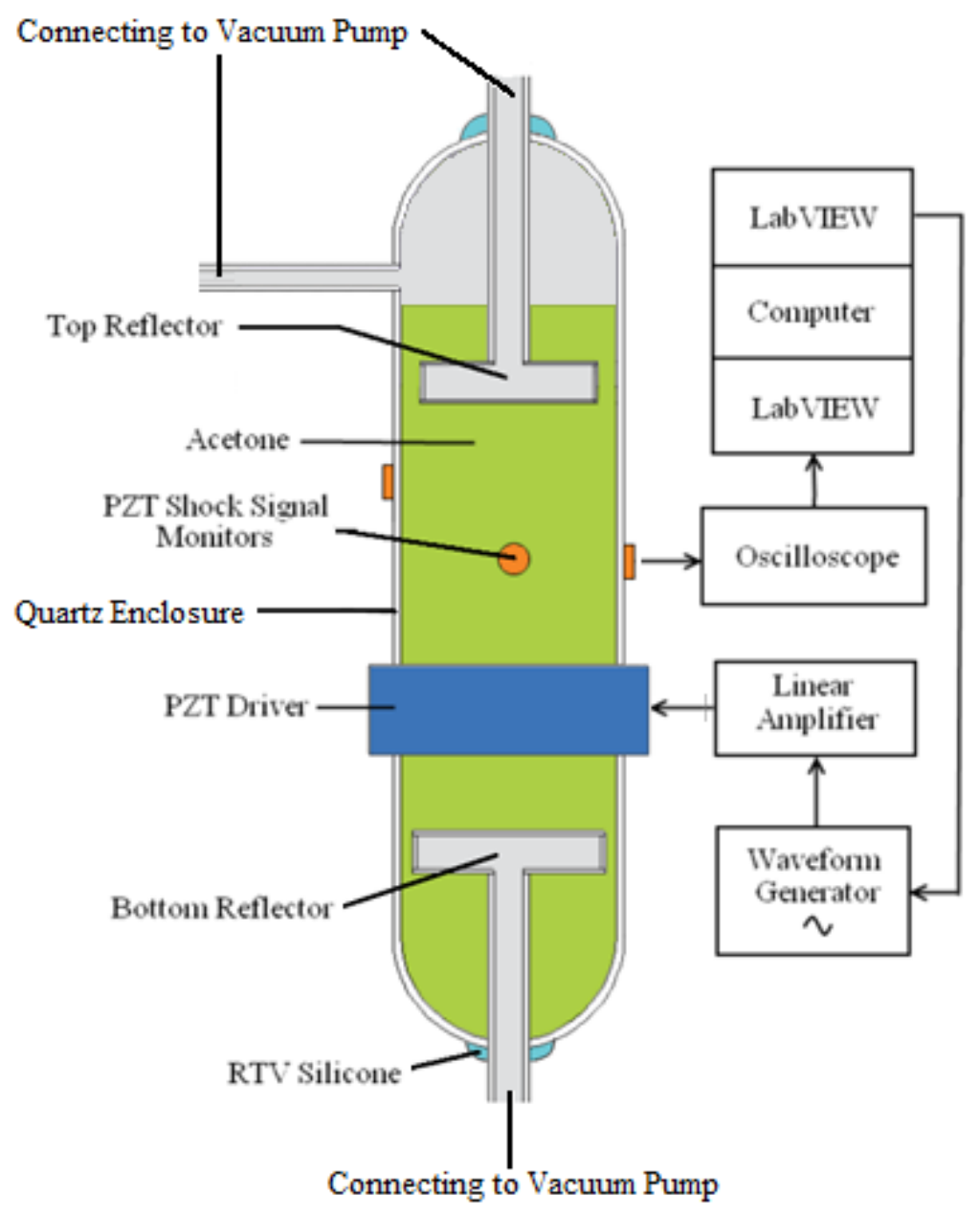

Figure 1. Schematic of cylindrical ATMFD system used for direction-position sensing studies.

During when the D-ATMFD fluid is under tension (below vacuum pressure), the state is metastable whereupon, neutron elastic scattering interactions may be monitored by the formation of transient bubbles [15][14]. Piezoelectric elements that are attached to the outside of the D-ATMFD are used to monitor for shock signals generated by the imploding cavitation bubbles which occurs immediately following a neutron induced detection event. The time difference of arrival (TDOA) of the shock signal at each transducer is measured and analyzed with a hyperbolic positioning algorithm to calculate the 3-D location of the neutron detection event in the D-ATMFD. Directional information is ascertainable in the D-ATMFD system due to 
52 the increased probability that a neutron induced detection event will occur in the region of the sensitive volume nearest the neutron source as opposed to the region of the sensitive volume furthest from the neutron source. It has been previously shown that the probability that a neutron induced detection event will occur is

a function of the negative pressure in the detector fluid and the energy and magnitude of the neutron flux [14]. Due to the axi-symmetric nature of the D-ATMFD, the probability of a neutron detection event in a given voxel can be simplified by treating it as a function of the neutron flux alone [16]. Uncoupling of the dependence of the probability of a neutron detection event with the negative pressure in the detector fluid allows one to quantify the $2 \pi$ directional capabilities of the D-ATMFD based solely on the magnitude and energy of the neutron flux [14]. Although the current study is focused on ascertaining directionality in $2 \pi$, alternate ATMFD geometries have been developed and proven capable of ascertaining directionality in full $4 \pi$ fields [12]. Since neutron flux reduces with distance and with the amount of down scattering and absorption in the detector fluid, the side of the sensitive volume nearest to the source naturally has the highest probability of neutron interaction locations, and therefore, for the formation of neutron induced detection events. Detecting the position of these events inside the detector now allows the user to ascertain information on the direction of the neutron source. This is accomplished via the stochastic directionality model developed previously [14] which utilizes the neutron attenuation law to estimate the probability, $P$, that a detected neutron had traversed a distance, $d$, in the detector fluid without interacting and then interacting within the distance $\delta d$ from the expression:

$$
P(d, \delta d)=P_{\text {non-reaction }}(d) \cdot P_{\text {reaction }}(\delta d)
$$

The distance, $d$, from any position in the detector to the wall of the detector in the direction, $\theta$, is derived as, 
$75 d=r \cos \left(\theta+\pi-\theta_{0}\right)+\sqrt{r^{2} \cos ^{2}\left(\theta+\pi-\theta_{0}\right)-r^{2}+R^{2}}$

\section{Refinement of the stochastic neutron directionality model for enhanced accuracy}

The stochastic directionality model as developed previously makes two basic assumptions: the effects of solid angle on the neutron flux are negligible (i.e. neutrons are traveling uni-directionally) and that the detected neutron traveled directly from the neutron source to the detection location (i.e. no interactions with 
96 the detector fluid occurred between the neutron detection location and the source origination). MCNP-

97 PoliMi assessments were performed to test the validity of these assumptions. Firstly, assessments were 98 performed with a ${ }^{252} \mathrm{Cf}$ fission neutron source at a direction of $0^{\circ}$, a distance of $50 \mathrm{~cm}$ from the center-line of 99 the ATMFD and a sensitive volume size of $\mathrm{r}=1.25 \mathrm{~cm}$ and $\mathrm{h}=4.0 \mathrm{~cm}$ (modeled as a right circular 100 cylinder). The locations of simulated neutron detection events in the sensitive volume of the detector were 101 then analyzed with the stochastic directionality model. The predicted source direction and angular resolution 102 at the $68 \%$ confidence level (C.L.) and 95\% C.L. were calculated for a sample size of 2000 simulated 103 detection events. The accuracy of the stochastic directionality model was calculated by testing whether the 104 105

true source direction (i.e. $0^{\circ}$ ) was within $\pm 1 \sigma$ and $\pm 2 \sigma$ of the predicted direction at the $68 \%$ C.L. and $95 \%$ C.L., respectively. Analysis of the simulated data sets revealed that the stochastic directionality model was $57.7 \%$ accurate at the $68 \%$ C.L. and $90.4 \%$ accurate at the $95 \%$ C.L. In order to test whether the deficient accuracy is the result of solid angle effects, MCNP-PoliMi assessments were performed utilizing a planar source geometry consisting of a uni-directional ${ }^{252} \mathrm{Cf}$ neutron source designed to eliminate the effects of solid angle and to simulate a source at an infinitely large standoff. As before, assessments were performed with a sensitive volume size of $\mathrm{r}=1.25 \mathrm{~cm}$ and $\mathrm{h}=4.0 \mathrm{~cm}$. The locations of simulated neutron detection events in the sensitive volume of the detector were then analyzed with the stochastic directionality model and the accuracy at $68 \%$ and $95 \%$ confidence levels were calculated. Analysis of the simulated data sets revealed that the stochastic directionality model is $52.9 \%$ accurate at the $68 \%$ C.L. and is $88.9 \%$ accurate at the $95 \%$ C.L. It is important to note that the accuracy actually increases (from $52.9 \%$ to $57.7 \%$ at the $68 \%$ C.L.) due to solid angle effects. Additional MCNP-PoliMi assessments were performed in order to test whether the reduced accuracy was due to the second assumption that the detected neutron traveled directly from the neutron source to the detection location (i.e. no interactions with the detector fluid occurred between the neutron detection location and the source origination). In order to investigate this possibility the percentage of neutrons that had scattered in the detector prior to depositing enough energy in a collision 
120 event to be detected were tallied. The assessments show that for a sensitive volume size of $\mathrm{r}=1.25 \mathrm{~cm}$ and $\mathrm{h}$ $121=4.0 \mathrm{~cm}, 41.2 \%$ of the detection events resulted from neutrons that had already scattered (i.e., did not 122 originate directly from the true neutron source direction). Assessments were then performed excluding these 123 events in order to quantify this effect on the predicted accuracy. Analysis of the simulated data sets revealed 124 that the stochastic directionality model is $69.8 \%$ accurate at the $68 \%$ C.L. and is $96.7 \%$ accurate at the $95 \%$ 125 C.L. This represents a substantial improvement and illustrates that the reduced accuracy is due primarily to 126 the scattering of neutrons prior to depositing enough energy in a collision event to be detected. Interestingly, 127 the improved accuracy does not come at the cost of degraded angular resolution, e.g., the angular resolution 128 at the $68 \%$ C.L. increased by $3.1^{\circ}$ to $\pm 9.9^{\circ}$ and by $6.2^{\circ}$ to $\pm 19.7^{\circ}$ at the $95 \%$ C.L. Unfortunately, 129 discriminating neutrons that had scattered before being detected is impossible in a real world application, 130 however, it is possible to account for this effect by calculating the angular resolution directly from the 131 performance of the stochastic directionality model.

132 This is accomplished by relating the length of the mean resultant vector to the angular resolution 133 achieved by the stochastic directionality model. This allows for the estimation of the angular resolution to 134 vary accordingly with the amount of directional information available. This was accomplished by utilizing 135 MCNP-PoliMi simulations in conjunction with the stochastic directionality model to calculate the 136 distribution in predicted source directions. This is done by redefining the predicted source direction as the mean angular direction, $\bar{\theta}$, of the total source direction probability which is derived as the phase of the mean resultant vector defined as,

$$
\bar{r}=\sum_{j=1}^{N} P_{\text {total }}\left(\theta_{j}\right) e^{i \theta_{j}}
$$


142 The calculation of the mean resultant vector allows for the calculation of the length of the mean resultant 143 vector, which is a quantity indicative of the circular spread of the angular source direction probability 144 distribution [17]. The length of the mean resultant vector, $R$, is calculated by,

$$
R=\frac{|\bar{r}|}{\sum_{j=1}^{N} P_{\text {total }}\left(\theta_{j}\right)}
$$

148 The relationship between the length of the mean resultant vector of the source direction probability 149 distribution and the angular resolution achieved forms the basis of the robust stochastic directionality model. 150 This relationship was determined by utilizing MCNP-PoliMi assessments performed with the ${ }^{239} \mathrm{Pu}-\mathrm{Be}$ neutron source simulated at a direction of $0^{\circ}$ and at a distance of $20.3 \mathrm{~cm}$ from the centerline of the $\mathrm{D}$ ATMFD. A sensitive volume size of $\mathrm{r}=1.25 \mathrm{~cm}$ and $\mathrm{h}=4.0 \mathrm{~cm}$ formed the volume for neutron tally simulations (equivalent to a $\sim 4 \mathrm{~W}$ drive power). The locations of neutron detection events in the D-ATMFD volume were then analyzed with the stochastic directionality model. Simulations were performed varying the sample size from 10 detection events to 3000 detection events. The prediction for the neutron source direction was then deemed to be located at the mean of the source direction probability distribution. The angular resolution at $68 \%$ C.L. was determined by calculating the $16^{\text {th }}$ and $84^{\text {th }}$ percentiles of the predicted source direction distribution. Similarly, the angular resolution at 95\% C.L. was determined by calculating the $2.5^{\text {th }}$ and $97.5^{\text {th }}$ percentiles of the predicted source direction distribution. The length of mean resultant vector, $R$, was calculated for each simulation, and the average length of the mean resultant vector for all simulations at each sample size was calculated. An example of the results of the MCNP-PoliMi assessments for a sample size of 100 detection events (left) and 1000 detection events (right) are shown in Figure 2. 

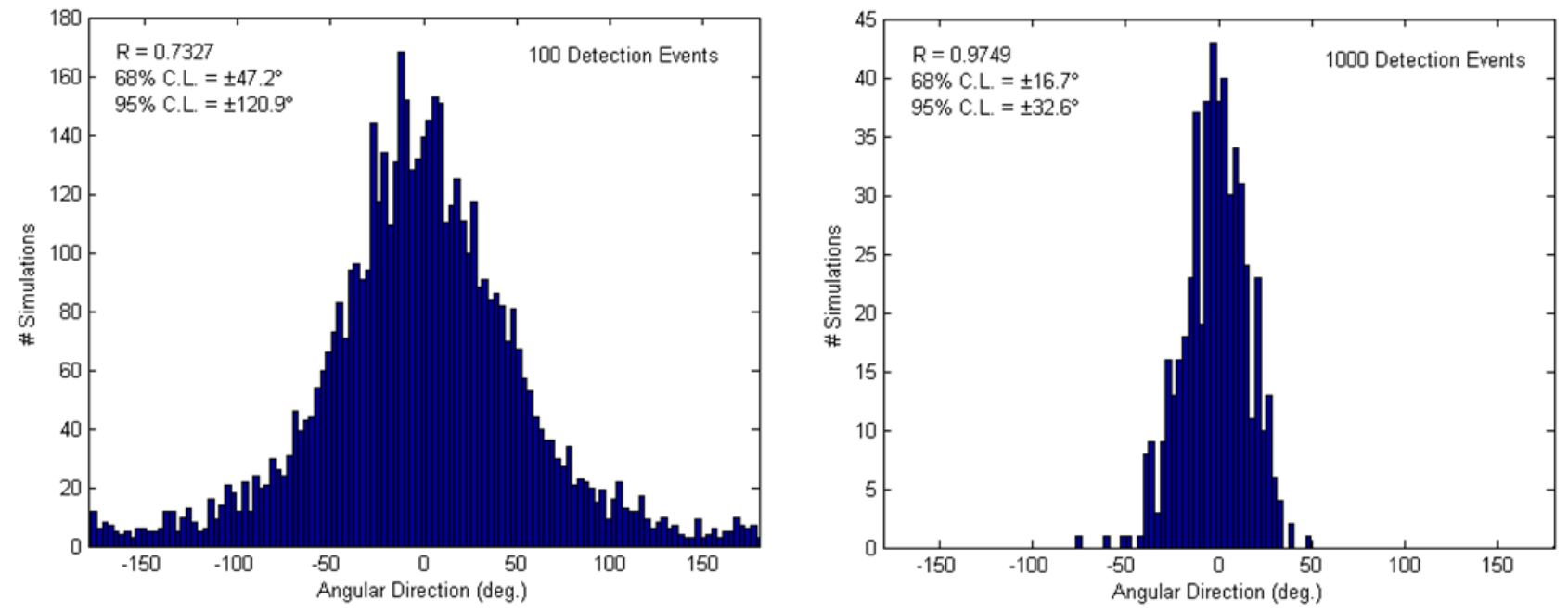

Figure 2. Distribution of mean source directions predicted by the stochastic directionality model. Assessments performed with MCNP-PoliMi with a SV size of $\mathrm{r}=1.25 \mathrm{~cm}$ and $\mathrm{h}=4$ $\mathrm{cm}$ with a $239 \mathrm{Pu}-\mathrm{Be}$ source at $0^{\circ}$ at a distance of $20.3 \mathrm{~cm}$. Examples shown for simulations with a sample size of 100 detection events (left) and 1000 detection events (right).

At a sample size of 100 detection events the angular resolution is $47.2^{\circ}$ and $120.9^{\circ}$ at the $68 \%$ C.L. and $95 \%$ C.L. respectively. The average length of the mean resultant vector was determined to be 0.7327 . For a sample size of 1000 detection events, the angular resolution is $16.7^{\circ}$ and $32.6^{\circ}$ at the $68 \%$ C.L and 95\% C.L. respectively. The average length of the mean resultant vector was determined to be 0.9749 . The results of all simulated sample sizes were compiled, and are shown in Figure 3. 


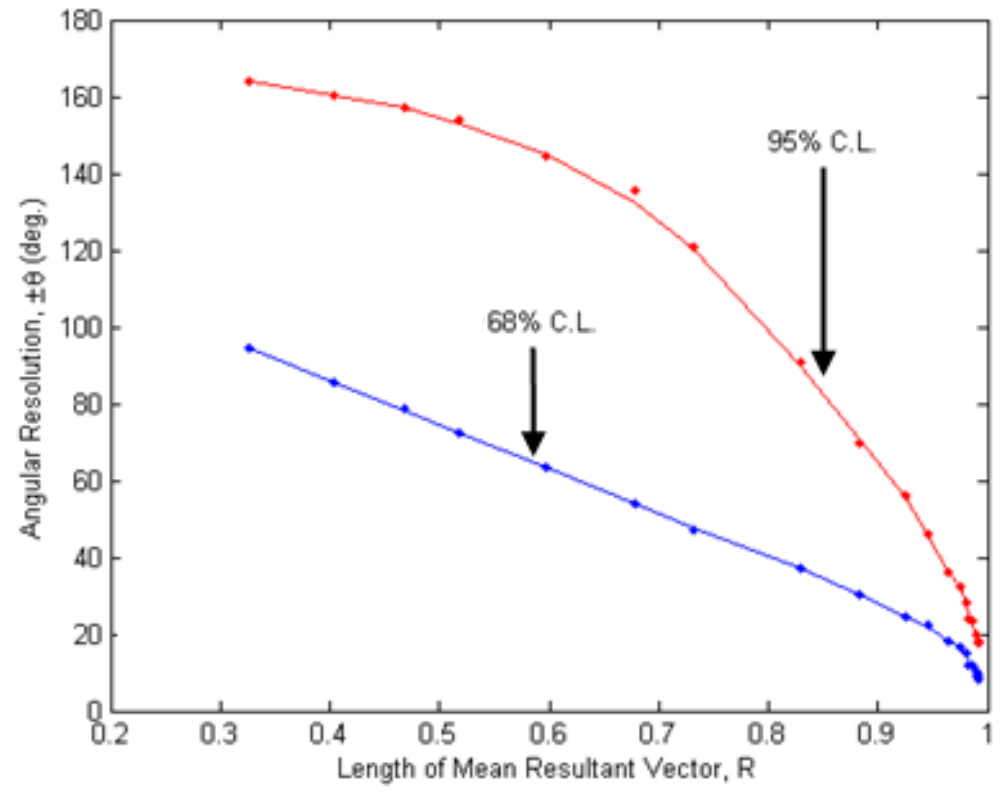

Figure 3. Determination of the angular resolutions at $68 \%$ C.L. and $95 \%$ C.L. as a function of the length of the mean resultant vector. Assessments performed with MCNP-PoliMi with a $\mathrm{SV}$ size of $\mathrm{r}=1.25 \mathrm{~cm}$ and $\mathrm{h}=4 \mathrm{~cm}$ with a $239 \mathrm{Pu}-\mathrm{Be}$ source at $0^{\circ}$ at a distance of $20.3 \mathrm{~cm}$.

Relating the angular resolution to the length of the mean resultant vector was accomplished by Lowess smoothing of the compiled data. This relationship forms the basis of the robust stochastic model, and allows the angular resolutions to be calculated accurately for each resultant source direction probability distribution.

In order to verify the validity of the robust stochastic model developed, MCNP-PoliMi assessments were performed with a sample size of 2000 detection events. The locations of simulated detection events pertaining to neutrons in the specific D-ATMFD volume were thereafter, assessed with the stochastic directionality model. The direction of the neutron source was predicted to be at the mean of the source direction probability distribution and the length of mean resultant vector, $R$, was calculated for each simulation. The length of the mean resultant vector was utilized to linearly interpolate the resultant angular resolution at the $68 \%$ C.L. and 95\% C.L (i.e. from the data shown in Figure 3). Validation included verifying the accuracy of the robust stochastic directionality model by testing whether the true source direction (i.e. $0^{\circ}$ ) was within the stated angular resolution of the predicted direction at the $68 \%$ C.L. and 95\% C.L. Analysis of a data set containing 262 individual simulations (each with a sample size of 2000 
194 detection events and $\mathrm{SV} r=1.25 \mathrm{~cm}$ and $\mathrm{h}=4.0 \mathrm{~cm})$ revealed that the stochastic directionality model is $19569.1 \%$ accurate at the $68 \%$ C.L. and is $95.8 \%$ accurate at the $95 \%$ C.L. This is substantial and represents a 196 significant improvement in accuracy provided by the robust stochastic directionality model when compared 197 to the stochastic directionality model previously developed (i.e. $69.1 \%$ vs. $53.8 \%$ at the $68 \%$ C.L. and $95.8 \%$ 198 vs. $88.6 \%$ at the $95 \%$ C.L.) $[14]$.

199 Experimentation was conducted to derive data using a $\sim 1 \mathrm{Ci}^{239} \mathrm{Pu}-\mathrm{Be}$ isotope neutron source providing $200 \sim 2 \times 10^{6}$ neutrons/s (equivalent to the intensity emitted from $\sim 3.5 \mathrm{~kg}$ of reactor grade plutonium [18]) 201 positioned at an angle of $0^{\circ}$ and distance of $\sim 80 \mathrm{~cm}$ at a D-ATMFD drive power of $\sim 4 \mathrm{~W}$. This resulted in 202

the formation of $\sim 10$ neutron induced detections per second resulting in the collection of $\sim 2,000$ neutron induced detection events which could be acquired within 200 seconds. Analysis of the locations of the neutron induced detection events with the robust stochastic directionality model determined the neutron source to be located at an angle of $5.6^{\circ}$ with an angular resolution of $\pm 13.8^{\circ}$ at a C.L. of $68 \%$, and $\pm 27.6^{\circ}$ at a C.L. of 95\%. These results demonstrated that the D-ATMFD system as used enabled locating the direction of the ${ }^{239} \mathrm{Pu}-\mathrm{Be}$ neutron source at a distance of $80 \mathrm{~cm}$ to within $\pm 13.8^{\circ}$ with $68 \%$ confidence within $\sim 200$ sec. (i.e., for a $\sim 1 \mathrm{Ci}^{239} \mathrm{Pu}-\mathrm{Be}$ neutron source at a distance of $80 \mathrm{~cm}$ ). This represents a substantial reduction (13 $\times$ ) in the search space when compared to a non-directional detector. The angular resolution previously calculated using the stochastic directionality model was $\pm 8.1^{\circ}$ at the $68 \%$ C.L., representing a decrease in angular resolution of $5.7^{\circ}[14]$. However, the robust stochastic directionality model developed resulted in an increase in the accuracy from $57.7 \%$ to $69.9 \%$ at the $68 \%$ C.L. As noted previously, as more and more neutron detection events are acquired, the angular resolution achievable with the D-ATMFD system increases dramatically. Figure 4 was prepared to demonstrate this effect. Notably, within only 10 seconds and via acquiring of only 100 neutron detection events using a single $600 \mathrm{~cm}^{3}$ D-ATMFD unit, the overall geometrical space for searching for the source has already been brought down by a factor of $\sim 6$. 


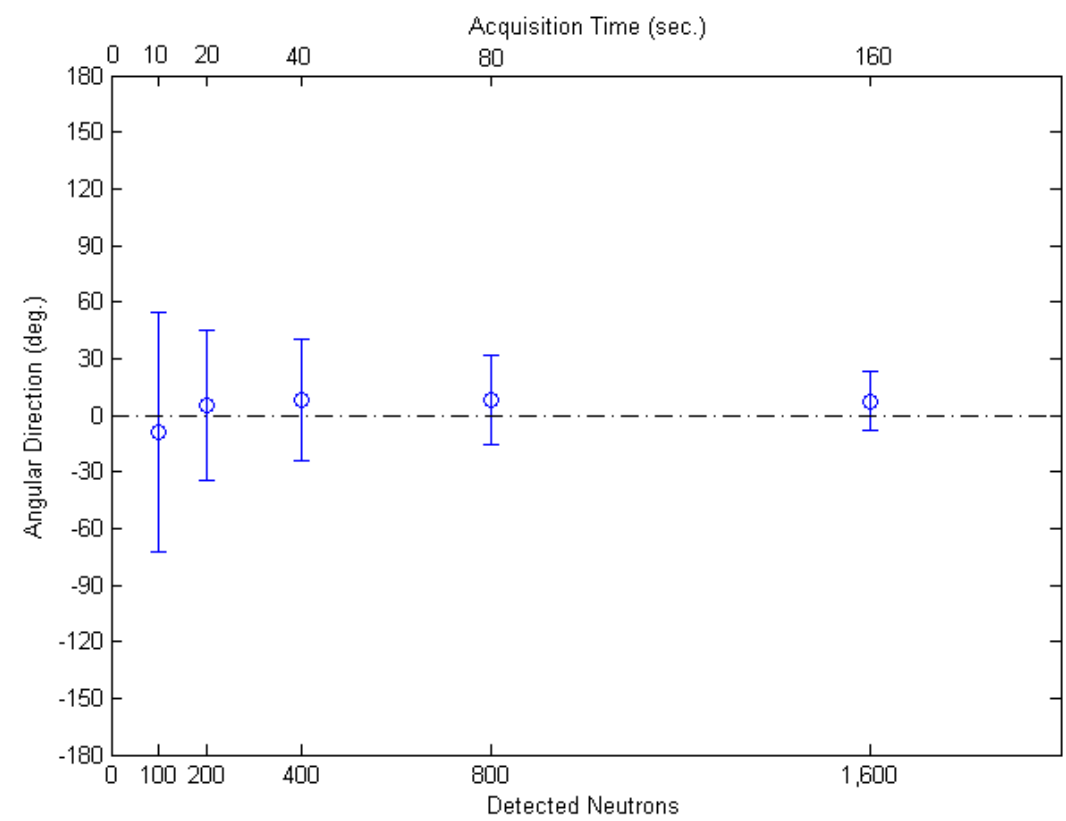

Figure 4. Depiction of precision for determining neutron radiation from a $1 \mathrm{Ci}^{239} \mathrm{Pu}-\mathrm{Be}$ neutron source located $80 \mathrm{~cm}$ from detector at $0^{\circ}$ improving dramatically with increased counts. Error bars pertain to $68 \%$ confidence. Time for acquisition was based on a detection rate of $\sim 10$ per second in the D-ATMFD.

Additionally, we also conducted experiments in which we acquired with a $\sim 45 \mu C i{ }^{252} \mathrm{Cf}$ isotope fission spectrum neutron source emitting $\sim 1.9 \times 10^{5}$ neutrons/s (equivalent to the intensity emitted from $\sim 2.5 \mathrm{~kg}$ of WGP [19]) positioned at $0^{\circ}$ and at about $20 \mathrm{~cm}$ away using a D-ATMFD drive power of $\sim 4 \mathrm{~W}$. This resulted in the formation of $\sim 5$ neutron induced detections per second subsequently resulting in $\sim 1,500$ neutron induced detection events which could be acquired within 300 seconds. Analysis of the experimental data with the robust stochastic directionality model determined the neutron source to be located at an angle of $0.4^{\circ}$ with data resolved to $\pm 11.4^{\circ}$ at a C.L. of $68 \%$, and $\pm 21.4^{\circ}$ at a C.L. of $95 \%$. This represents an improvement in the angular resolution (i.e. $2.4^{\circ}$ better at the $68 \%$ C.L. and $6.2^{\circ}$ better at the $95 \%$ C.L) achievable with the robust stochastic directionality model when ascertaining the direction of a ${ }^{252} \mathrm{Cf}$ isotope fission spectrum neutron source and required $\sim 25 \%$ fewer detection events. This is substantial and represents a corresponding $\sim 25 \%$ decrease in the time to detect the direction of a SNM neutron source. The increased directional precision is due to the higher elastic scattering cross section (and consequently 
235 increased down scattering and absorption in the detector fluid) for neutrons from the spontaneous fission of $236{ }^{252} \mathrm{Cf}$.

237

238

\section{Scaling of D-ATMFDs for optimization of directionality information}

As mentioned previously, the neutron flux as measured by the D-ATMFD is a function of the distance from the source, and the level of scattering and absorption occurring in the detector fluid. Therefore, a detector with a larger sensitive volume is sensitive to a greater amount of neutron down scattering and absorption and consequently an increased amount of directional information is available. Previous work has shown via COMSOL multi-physics based assessments and direct experimental measurements of the distribution density of neutron-induced bubble nucleation sites that the size of the sensitive volume (and consequently the radius) is directly related to the input power of the D-ATMFD resonant chamber. For example, a relatively small $(\sim 40 \%)$ rise in the input power from $4 \mathrm{~W}$ to $5.5 \mathrm{~W}$ resulted in significant increase of the sensitive volume (by $\sim 75 \%$ ), i.e., from $\sim 20 \mathrm{~cm}^{3}$ to $\sim 35 \mathrm{~cm}^{3}[14][15]$. This is based on the principle that the higher the negative pressure amplitude in the detection fluid is, the higher the probability that a neutron induced detection event will occur. The increase in the amount of directionality information attainable with the robust stochastic directionality model was assessed via MCNP-PoliMi simulations. Several simulations were performed with various detector sensitive volume sizes ranging from a radius of $0.5 \mathrm{~cm}$ to $3.0 \mathrm{~cm}$ with a fixed height of $4.0 \mathrm{~cm}$ (sensitive volume approximated as a right circular cylinder tally volume). The locations of simulated neutron detection events occurring were then analyzed with the robust stochastic directionality model and the accuracy at $68 \%$ and $95 \%$ confidence levels were calculated.

The results are shown in Figure 5. These results illustrate that as the drive power and subsequently the radius of the sensitive volume increase, the resolution that may be achieved increases significantly due to not only an increased sensitive volume size, but also simultaneously due to improved detection efficiency. These results provide proof that the D-ATMFD capabilities are amenable for enhancement and possible to 


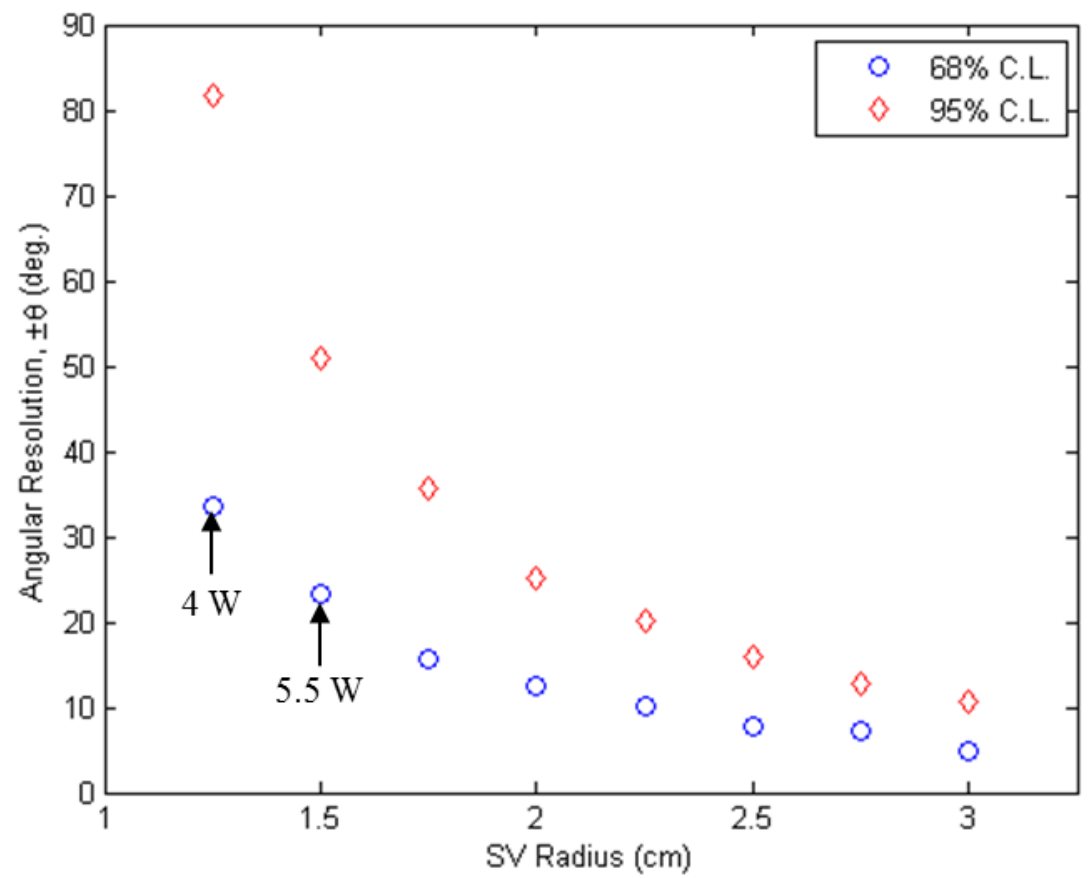

Figure 5. Pointing accuracy of the D-ATMFD increases with the increased sensitive volume radius and increased detection efficiency. MCNP-PoliMi simulations of D-ATMFD were performed until 500 neutron detection events were recorded for $\mathrm{R}=1.25 \mathrm{~cm}$ case. Number of detection events used for other cases are scaled appropriately. All cases used a SV height of $4.0 \mathrm{~cm}$.

\section{The D-ATMFD used for localizing the neutron source -- experiments and analysis}

An example of one of the applications of a directional-capable neutron detector system is the ability to image a neutron source which allows for the determination of the neutron source shape, size and strength. Neutron imaging detectors thus have the potential to play an important role in non-proliferation type applications. Localization of the neutron source is accomplished by utilizing multiple detectors that are located such that the angle subtended by the detector and the direction of the source is distinct for each detector. Experimentation was performed to investigate capabilities of the D-ATMFD system for this type of application. Two D-ATMFD detectors (each operated at a drive power of $\sim 4 \mathrm{~W}$ ) were placed $25 \mathrm{~cm}$ apart (as measured from the centerline of each D-ATMFD). The XY coordinate system was defined such that the detectors were located at $(0 \mathrm{~cm}, 0 \mathrm{~cm})$ and $(25 \mathrm{~cm}, 0 \mathrm{~cm})$ and a $2 \mathrm{D}$ mesh was created covering an 
experimental area of $1 \mathrm{~m}^{2} . \mathrm{A} \sim 1 \mathrm{Ci}^{239} \mathrm{Pu}-\mathrm{Be}$ neutron source was placed at $(15 \mathrm{~cm},-20 \mathrm{~cm})$ and data were recorded. This enabled collecting $\sim 2,000$ neutron induced detection events within $\sim 200$ seconds.

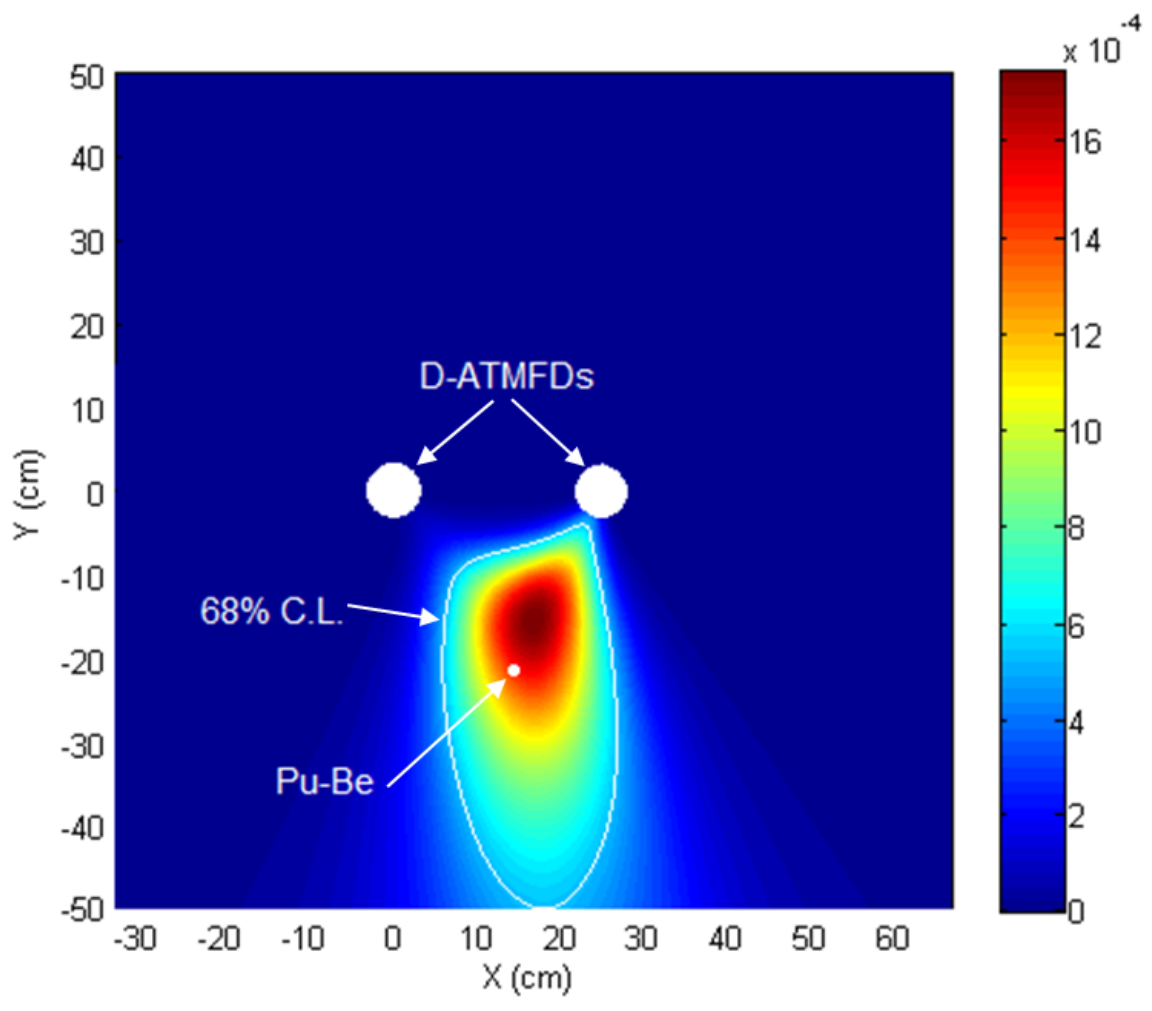

Figure 6. Experimentally measured and calculated neutron source location probability. ${ }^{239} \mathrm{Pu}-$ Be neutron source located at $(15 \mathrm{~cm},-20 \mathrm{~cm})$. The contour scale is in units of source location probability density per unit area $\left(\mathrm{mm}^{2}\right)$.

The locations of simulated neutron detection events were thereafter, assessed using the robust stochastic directionality model to evaluate the total source direction probability distribution function for each detector and then the corresponding total source location probability was calculated. The results are shown in Figure

6. The neutron source was determined to be located at the most probable location of $(16.6 \mathrm{~cm},-15.6 \mathrm{~cm})$ and the confidence level was calculated by directly integrating the area under the surface and was found to cover an area of $750 \mathrm{~cm}^{2}$ at a $68 \%$ confidence level. Additionally, a MCNP-PoliMi model was developed to simulate the experimental conditions. The experimental conditions were replicated in the model, and simulations were run until a total of 2000 neutron detection events occurred in the D-ATMFDs. The neutron source was determined to be located at the most probable location at $(18 \mathrm{~cm},-17.4 \mathrm{~cm})$. The confidence 
291 level was calculated by integrating the area under the surface and was found to cover an area of $1020 \mathrm{~cm}^{2}$ at $29268 \%$ confidence. Importantly, both MCNP-PoliMi simulations and experimental data resulted in very 293 similar neutron source locations, and the true source location was well within the predicted $68 \%$ confidence 294 level for both cases. Comparing these results to the previously published values for a similar experiment 295 performed indicate that the assumptions previously made neglecting multiple scattering and solid angle 296 effects resulted in an overestimation of the confidence. This was expected as the neutron source was located 297 at a distance that was sufficiently close enough that the effects of solid angle were not negligible.

\section{D-ATMFD based ANSI Cat. E RPM Simulations}

Another example for demonstrating one of many unique applications of the D-ATMFD neutron detector is the external non-intrusive monitoring and imaging of moving neutron sources, including special nuclear material, in containers and packages being scanned. The design of one such D-ATMFD based radiation detection portal monitor was assessed following ANSI standard N42.35-2006 which is intended to provide the testing and evaluation criteria for installed radiation detection portal monitors that detect radioactive materials (without identification capabilities) [21]. The standard outlines performance criteria and test methods used to evaluate installed radiation detection portal monitors (RPM) for use in U.S. Homeland Security. The standard states that the source shall be moved at a specified speed dependent on the type of monitor. The setup or evaluation distances vary for different applications, in which the distances are measured from the face of the detector case or assembly. The baseline case we studied was targeted for a category E configuration representing a multiple-sided pedestrian or package monitor. The D-ATMFD based RPM simulated consists of 4 separate panels $(20 \mathrm{~cm} \times 20 \mathrm{~cm} \times 60 \mathrm{~cm})$, each with 6 individual DATMFD elements with a sensitive volume of $\mathrm{r}=3.0 \mathrm{~cm}$ and $\mathrm{h}=5.0 \mathrm{~cm}$. The spacing between D-ATMFD panels along the axis of the conveyer was $1 \mathrm{~m}$ as measured from the center-line of each panel. The detectors are separated $1 \mathrm{~m}$ apart as measured from the external face of the detector assembly. The detector fluid simulated was acetone. An illustration of the simulated D-ATMFD based RPM is shown in Figure 7. 


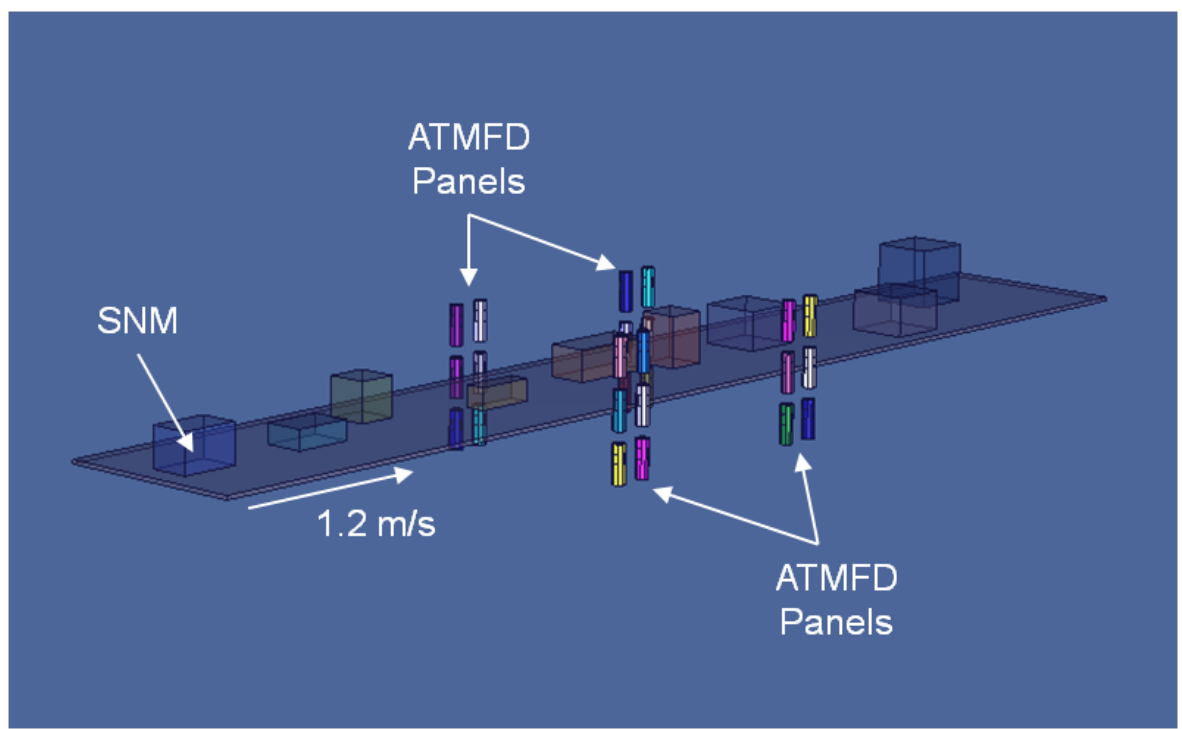

Figure 7. Schematic of D-ATMFD based ANSI Cat. E. RPM system consisting of 24 D-

ATMFD elements.

318 The source speed was set at $1.2 \mathrm{~m} / \mathrm{s}$. Preliminary assessments simulated a ${ }^{252} \mathrm{Cf}$ based neutron source with 319 an activity equivalent to an $4 \mathrm{~kg}$ mass of weapons grade plutonium in a metallic sphere $\left(\sim 1 \times 10^{6} \mathrm{n} / \mathrm{s}\right)[19]$. 320 The simulation is started with the SNM source located at the center of the conveyer and $3 \mathrm{~m}$ away from the 321 center-line of the D-ATMFD RPM assembly. The locations of simulated neutron detection events are 322 analyzed as they occur in the simulation with the robust stochastic directionality model in order to calculate 323 the source location probability distribution. An example of the source location probability distribution 2 324 seconds into a simulation is shown in Figure 8. The location of the SNM source was correctly identified to 325 within $0.2 \mathrm{~m}^{2}$ with $68 \%$ confidence within 2 seconds. The acquisition of additional neutron detection events allows for further refinement of the location of the SNM source. After 4.75 seconds, the location of the SNM source was correctly identified to within an area of $0.06 \mathrm{~m}^{2}$ (see Figure 9). 


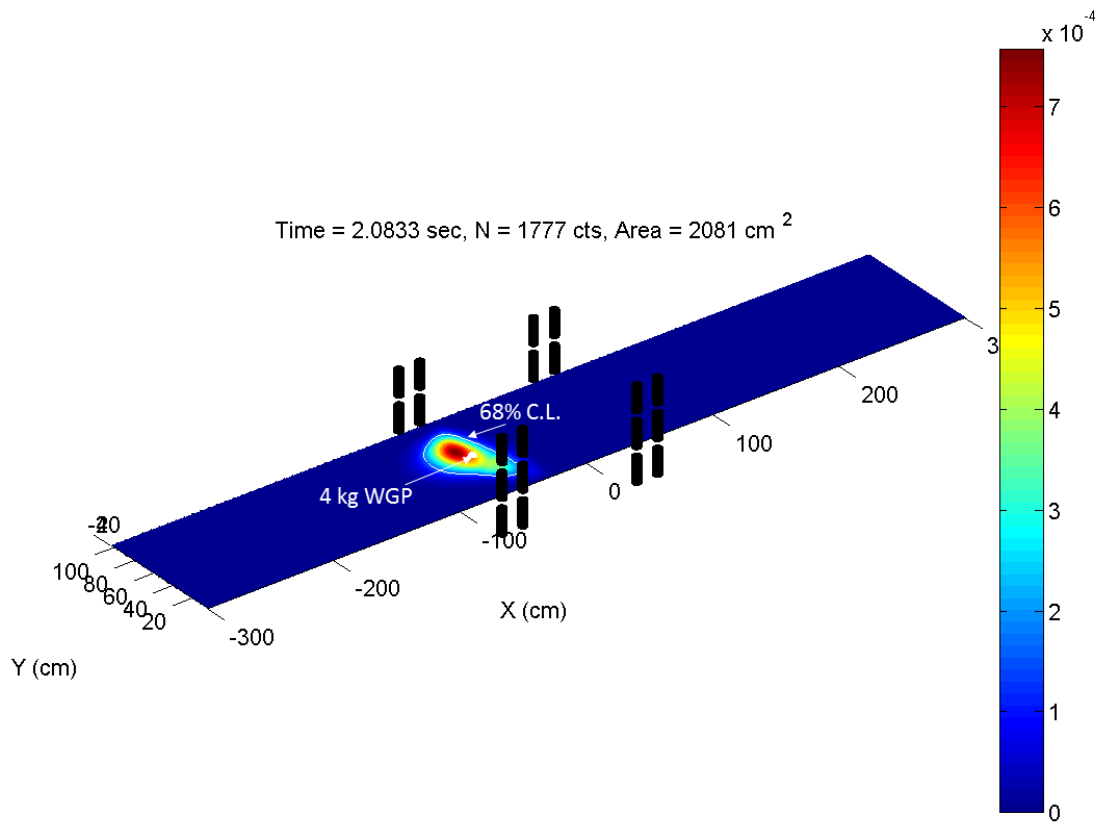

Figure 8. Simulation of the passage of an $4 \mathrm{~kg}$ mass of WGP thru the D-ATMFD based RPM. Figure shown taken at $\mathrm{T}=2$ seconds. The contour scale is in units of source location probability density per unit area $\left(\mathrm{cm}^{2}\right)$.

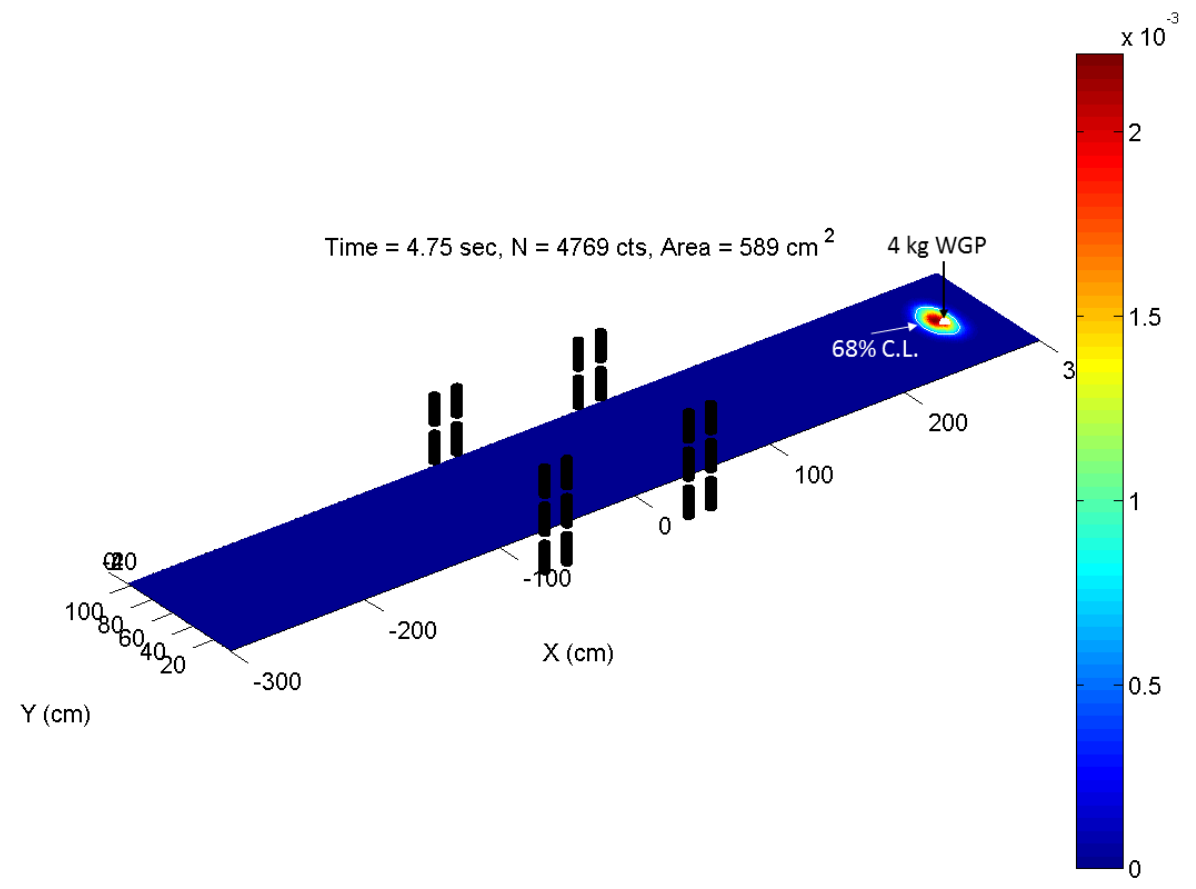

Figure 9. Simulation of the passage of an $4 \mathrm{~kg}$ mass of WGP thru the D-ATMFD based RPM. Figure shown at $\mathrm{T}=4.75$ seconds. The contour scale is in units of source location probability density per unit area $\left(\mathrm{cm}^{2}\right)$. 
336 Additional assessments included a ${ }^{252} \mathrm{Cf}$ based neutron source with an activity equivalent to a $2 \mathrm{~kg}$ of weapons grade plutonium $\left(\sim 1.2 \times 10^{5} \mathrm{n} / \mathrm{s}\right)$ [19]. As before, the simulation is started with the SNM source located at the center of the conveyer and $3 \mathrm{~m}$ away from the center-line of the D-ATMFD RPM assembly.

The locations of simulated neutron detection events are analyzed as they occur in the simulation with the robust stochastic directionality model in order to calculate the source location probability distribution. The location of the SNM source was correctly identified to within an area of $0.3 \mathrm{~m}^{2}$ with $68 \%$ confidence within 5 seconds.

\section{Conclusions and Future Work}


source, an aspect which was validated via experimentation, and also successfully simulated with a MCNP-

PoliMi modeling scheme. A D-ATMFD based RPM capable of the external non-intrusive monitoring and imaging of sources of fast neutrons, including SNM, in containers and packages was developed and characterized. The D-ATMFD based RPM developed was shown to be capable of ascertaining the location of a $4 \mathrm{~kg}$ mass of WGP moving at a speed of $1.2 \mathrm{~m} / \mathrm{s}$ to within $0.06 \mathrm{~m}^{2}$ in less than 5 seconds. Planned work includes assessing the possibility of further enhancing directional and positional information via higher drive powers, higher sensitivity detection fluids, and potential physical scaling of the detector size, and to investigate additional nuclear security safeguards applications.

\section{Acknowledgment}

The reported work for this paper was sponsored by Sagamore Adams Laboratories, LLC and is gratefully acknowledged. This work builds upon past work supported in part by funds from other sponsors such as the U.S. Department of Defense, the U.S. Department of Energy, the U.S. National Science Foundation, and the Department of Homeland Security. This work also benefited from assistance from Purdue University’s REM and several other Purdue staff and are acknowledged.

\section{References}

[1] S. Ahlen, D. Dujmic, P. Fisher, A. Inglis, H. Tomita, and H. Wellenstein, "A Background-Free Direction-Sensitive Neutron Detector," IEEE Trans. Nucl. Sci., vol. 57, no. 5, pp. 2740-2746, Oct. 2010.

[2] N. S. Bowden, M. Heffner, G. Carosi, D. Carter, P. OMalley, J. Mintz, M. Foxe, I. Jovanovic, and P. O’Malley, "Directional fast neutron detection using a time projection chamber," Nucl. Instruments Methods Phys. Res. Sect. A Accel. Spectrometers, Detect. Assoc. Equip., vol. 624, no. 1, pp. 153-161, Dec. 2010.

[3] N. Mascarenhas, W. Mengesha, J. Peel, and D. Sunnarborg, "Directional Neutron Detectors For Use with $14 \mathrm{MeV}$ Neutrons," 2005.

[4] W. Mengesha, N. Mascarenhas, J. Peel, D. Sunnarborg, A. The, and G. Monte, "Modeling of a Directional Scintillating Fiber Detector for $14 \mathrm{MeV}$ Neutrons," vol. 53, no. 4, pp. 2233-2237, 2006.

[5] N. Mascarenhas, J. Brennan, K. Krenz, P. Marleau, and S. Mrowka, "Results With the Neutron Scatter Camera," IEEE Trans. Nucl. Sci., vol. 56, no. 3, pp. 1269-1273, Jun. 2009.

[6] U. Bravar and P. Bruillard, "Design and testing of a position-sensitive plastic scintillator detector for fast neutron imaging,” Nucl. Sci. ..., vol. 53, no. 6, pp. 3894-3903, 2006. 
[7] G. a. de Nolfo, S. D. Hunter, J. T. Link, and N. a. Guardala, "Neutron Imaging Camera," 2009 IEEE Conf. Technol. Homel. Secur., pp. 282-286, May 2009.

[8] R. S. Woolf, P. F. Bloser, U. Bravar, J. S. Legere, M. L. McConnell, J. R. Macri, P. C. Mallik, B. Pirard, J. M. Ryan, and J. R. Wood, "Imaging and spectroscopy of fission neutrons with the FNIT experiment," 2009 IEEE Conf. Technol. Homel. Secur., pp. 274-281, May 2009.

[9] E. Fenimore and T. Cannon, "Coded aperture imaging with uniformly redundant arrays," Appl. Opt., pp. 1-9, 1978.

[10] P. Marleau, J. Brennan, E. Brubaker, and J. Steele, "Results from the coded aperture neutron imaging system," IEEE Nucl. Sci. Symp. Med. Imaging Conf., pp. 1640-1646, Oct. 2010.

[11] R. S. Woolf, B. F. Phlips, A. L. Hutcheson, L. J. Mitchell, and E. a. Wulf, "An active interrogation detection system (ACTINIDES) based on a dual fast neutron/gamma-ray coded aperture imager," 2012 IEEE Conf. Technol. Homel. Secur., pp. 30-35, Nov. 2012.

[12] B. Archambault, T. F. Grimes, J. A. Webster, N. W. Wilson, A. Hagen, K. Fischer, and R. P. Taleyarkhan, "Development of a $4 \pi$ directional fast neutron detector using tensioned metastable fluids," 2012 IEEE Conf. Technol. Homel. Secur., pp. 423-428, 2012.

[13] T. F. Grimes, B. Archambault, J. a. Webster, A. Sansone, and R. P. Taleyarkhan, "Gamma-blind transformational nuclear particle sensors," 2012 IEEE Conf. Technol. Homel. Secur., no. 1, pp. 417-422, Nov. 2012.

[14] B. C. Archambault, J. A. Webster, J. R. Lapinskas, T. F. Grimes, and R. Taleyarkhan, "Development of a novel directionposition sensing fast neutron detector using tensioned metastable fluids," Nucl. Instruments Methods Phys. Res. Sect. A Accel. Spectrometers, Detect. Assoc. Equip., vol. 673, pp. 89-97, May 2012.

[15] R. Taleyarkhan, J. Lapinskas, and Y. Xu, "Tensioned metastable fluids and nanoscale interactions with external stimuliTheoretical-cum-experimental assessments and nuclear engineering applications," Nucl. Eng. Des., vol. 238, no. 7, pp. 1820-1827, Jul. 2008.

[16] J. Wang and B. C. Archambault, "Numerical simulation and experimental study on Resonant Acoustic Chambers - For novel, high-efficiency nuclear particle detectors," in Proceedings of the 17th International Conference on Nuclear Engineering, 2010, pp. 1-11.

[17] J. Zar, Biostatistical Analysis, 5th ed. Prentice Hall, Inc., 2009.

[18] V. Bom, "Fast Neutron Imaging for SNM Detection," in Proceedings of the International Topical Meeting on ..., 2009, no. table 1, pp. 1-7.

[19] R. T. Kouzes, E. R. Siciliano, J. H. Ely, P. E. Keller, and R. J. McConn, "Passive neutron detection for interdiction of nuclear material at borders," Nucl. Instruments Methods Phys. Res. Sect. A Accel. Spectrometers, Detect. Assoc. Equip., vol. 584, no. 2-3, pp. 383-400, Jan. 2008.

[20] R. C. Runkle, "Neutron sensors and their role in nuclear nonproliferation," Nucl. Instruments Methods Phys. Res. Sect. A Accel. Spectrometers, Detect. Assoc. Equip., vol. 652, no. 1, pp. 37-40, Oct. 2011.

[21] ANSI, "American National Standard for Evaluation and Performance of Radiation Detection Portal Monitors for Use in Homeland Security Accredited by the American National Standards Institute," Washington, D.C., 2006. 